\title{
The importance of isolated patches for maintaining local bird biodiversity and ecosystem function: a case study from the Pernambuco Center of Endemism, Northeast Brazil
}

\author{
Leila F. A. S. Campos (1), Bruno P. Teixeira \& Márcio A. Efe (1)
}

Laboratório de Bioecologia e Conservação de Aves Neotropicais - ICBS, Universidade Federal de Alagoas, Campus A. C. Simões, Av. Lourival de Melo Mota, km 14, Cidade Universitária, 57072-970 Maceió, AL. (marcio_efe@yahoo.com.br)

\author{
Received 7 August 2017 \\ Accepted 8 April 2018 \\ Published 21 June 2018 \\ DOI: 10.1590/1678-4766e2018021
}

ABSTRACT. The Atlantic Forest has been highly fragmented, with the Pernambuco Center of Endemism (PCE) one of the priority areas for conservation. The Mata do Cedro forest, located in Alagoas state, northeastern Brazil, is a forest fragment within the PCE surrounded by a matrix of sugarcane that acts as a refuge for several threatened bird species, some of which are endemic to the region. Here, we characterize the bird community in Mata do Cedro using measures of species abundance, frequency of occurrence, habitat use and sensitivity to human disturbance. The functional role of species was investigated with a functional dendrogram. We registered 111 species, most resident and forest dependent. The most representative trophic categories were insectivores followed by frugivores. Of the species found, 11 are highly sensitive to human disturbances and 11 are endemic to the PCE. The bird community of the fragment is highly diversified, with endemic taxa and balanced trophic categories typical of preserved tropical forests. This community structure together with the occurrence of threatened species reinforces the importance of Mata do Cedro for the maintenance of local biodiversity and ecosystem functions.

KEYWORDS. Atlantic Forest, fragmentation, functional groups, community structure, Alagoas.

RESUMO. A importância dos fragmentos isolados para a manutenção da avifauna local e funções ecossistêmicas: caso de estudo no Centro de Endemismo Pernambuco, Nordeste do Brasil. A Mata Atlântica está altamente fragmentada, sendo o Centro de Endemismo Pernambuco (CEP) uma das áreas prioritárias para a conservação. A Mata do Cedro, localizada no estado de Alagoas, nordeste do Brasil, é um fragmento florestal do CEP rodeado por uma matriz de cana-de-açúcar que atua como refúgio para várias espécies de aves ameaçadas, algumas das quais são endêmicas da região. Este estudo caracterizou a comunidade de aves usando medidas de abundância de espécies, frequência de ocorrência, uso do habitat e sensibilidade aos distúrbios humanos. O papel funcional das espécies foi investigado com um dendrograma funcional. Nós registramos 111 espécies, sendo a maioria residente e dependente de florestas. As categorias tróficas mais representativas foram insetívoros, seguidas por frugívoros. Das espécies registradas, 11 são altamente sensíveis aos distúrbios humanos e 11 são endêmicas do CEP. A comunidade de aves do fragmento é altamente diversificada, com táxons endêmicos e categorias tróficas balanceadas típicas de florestas tropicais conservadas. Esta estrutura da comunidade, juntamente com a ocorrência de espécies ameaçadas, reforça a importância da Mata do Cedro para a manutenção da biodiversidade local e funções ecossistêmicas.

PALAVRAS-CHAVE. Mata Atlântica, fragmentação, grupos funcionais, estrutura de comunidade, Alagoas.

Birds are key players in ecosystems, maintaining crucial ecosystem functions, such as predation, pollination, ecosystem engineering and seed dispersion (SEKERCIOGLU et al., 2004; SeKercioglu, 2006; Whelan et al., 2008). However, in highly fragmented habitats a large number of diet specialized species, such as large frugivores and understory insectivores, perish (ANTUNES, 2005). In addition, changes in solar radiation, humidity and wind pattern associated with fragmentation are important for many organisms, as well as increases of edge habitats (RANTA et al., 1998). Furthermore, the degree of isolation between fragments can reduce genetic variability of populations and cause functional homogenization, restricting the quality of those fragments throughout time (CLAVEL et al., 2010).
Indeed, functional structure of bird communities has emerged as one of the key aspects to be considered in studies of habitat fragmentation (GIRAO et al., 2007; CADOTTE et al., 2011). Habitat fragmentation may lead to species loss from smaller fragments (SHAFFER, 1981) and, since highly specialized species are generally the most vulnerable to local extinction (SHULTZ et al., 2005), functional structure analysis may provide a measure of patche quality. Using this concept, is therefore possible to quantify the impact of fragmentation on species in a given area based on the ecological functions they mediate (GRIFFIN et al., 2009).

The Brazilian Atlantic Forest is one of the most threatened biomes in the world due to a long history of deforestation and degradation (Sнімамото et al., 2014). 
In the Northeast region, one particular area known as the Pernambuco Center of Endemism (PCE) (SILva et al., 2004 ) is both biologically rich and highly degraded (SILVA $\&$ TABARELLI, 2000). The PCE contains only $2 \%$ of its original vegetation cover and also suffers from high levels of illegal hunting and trapping of wildlife (SILVA \& TABARELLI, 2000; SILVEIRA et al., 2003).

In this study, we aim to characterize functional structure of a bird community of a fragmented area of the PCE.

\section{MATERIAL AND METHODS}

Study area. The Mata do Cedro Forest fragment $\left(09^{\circ} 31^{\prime} \mathrm{S}, 35^{\circ} 54^{\prime} \mathrm{W}\right)$ is situated within the Pernambuco Center of Endemism (PCE), in the municipality of Rio Largo, Alagoas State, northeast Brazil. It is characterized as open ombrophilous forest and covers approximately 1000 ha (PEReira et al., 2014, 2016) surrounded by sugar cane monocultures. Inside the forest there are several clearings undergoing regeneration and a network of wide trails. The middle stratum and canopy have several species of epiphytes and lianas.

Bird Sampling. Bird surveys were conducted from March 2010 to September 2011 using count points (Vielliard \& Silva, 1990), with adaptations suggested by VERGARA et al. (2010). Ten minutes were spent in each point with 2-5 minutes waiting period before starting the recording of contacts to minimize the disturbance of the arrival of the observer to the point. Eighteen points $200 \mathrm{~m}$ equidistant from each other were previously selected from preexisting track. Distance between the points was measured with GPS.

During sampling all audio and visual contacts were recorded. The visual contacts were obtained through direct observation with binoculars. The identifications were made based on a field guide (SIGRIST, 2009). Vocalizations were recorded with a Sony digital recorder and Yoga directional microphone. Recordings were later analyzed and compared with those available from electronic databases (http://www. xeno-canto.org/). The playback method was used with the aid of an amplifier box and the unknown individual recording vocalization was reproduced in order to lure the animal and make a visual identification.

Samples taken between March 2010 to November 2010 did not follow a standard in sampling effort and served to widen the qualitative survey and help familiarize the researcher with the local avifauna. Samples taken between December 2010 and September 2011 were standardized, so that 10 points were sampled at each visit, drawn from the 18 previously selected points. To obtain the total species richness the records of the entire study ( 14 visits $=70$ hours $)$ were considered, at listening points and outside of these (in moving from one point to another or on active pursuits).

Analysis. A Spot Index of Abundance (IPA) following VIELLIARD \& Silva (1990) was calculated to describe the abundance of each species, considering data from eight visits in the period December 2010 to September 2011 (13.33 hours). The degree of heterogeneity of the area based on proportional abundance of all species in the community was evaluated using the Shannon index. Pielou's evenness index was used to verify the relationship between the diversity observed and the maximum possible diversity for the same number of species. This index was calculated with data from eight visits conducted from December 2010 to September 2011.

The frequency of occurrence (FO) was based on the number of visits, expressed as a percentage, in which each species appeared (VIELlIARD \& SILVA 1990). Species with FO greater than or equal to $75 \%$ are considered residents and abundant (AleiXo \& Vielliard, 1995; AlmEIDA et al., 1999). Each species was assigned a category of abundance following AlmeIDA et al. (1999): (a) Resident: FO species with over $50 \%$; (b) Occasional: species living in other environments that occasionally exploit a feature of the forest; (c) Wandering: species of irregular occurrence in the woods or that were found only once; (d) Migrating: migratory species.

Regarding habitat use, species were classified based on information obtained from the field, complemented by Ridgely \& Tudor (1989, 1994), Silva (1995), PARKer et al. (1996) and SicK (1997), as forest independent, forest semi-dependent and forest dependent.

Taxa were grouped on their trophic categories according to TRAYLOR \& FitZPATRICK (1982), TERBORGH $e t$ al. (1990), Ridgely \& Tudor (1989, 1994) and Sick (1997). Preferred foraging stratum (soil, intermediate, superior or vertical) were based on DonATELLi et al. (2004) and SicK (1997). Sensitivity to human disturbance was assessed by placing species in one of three ordinal categories (high; average and low) following PARKer et al. (1996). The software Analytic Rarefaction 1.3 (HoLland, 2003) was used to calculate the cumulative rarefaction species curve. Based on each species registered (rows) and its respective functional traits (columns), a matrix was constructed for obtaining a functional dendrogram using R software, Picante and Vegan packages (R CoRE TEAm, 2014). An adaptation of the Gower Distance (PAvoIne et al., 2009) was used to calculate similarity. The Unweighted Pair Group Method with Arithmetic Mean (UPGMA) algorithm (SOKAL \& MicHENER, 1958) was used for constructing dendrograms and clustering.

Scientific nomenclature follows the recommendations of the Brazilian Ornithological Records Committee (Piacentini et al., 2015). Endemic species of the PCE were identified based on PEREIRA et al. (2016) and endemic Atlantic Forest species were based on CRACRAFT (1985). The classification of endangered species and their categories was based on the Brazilian Red List (MMA, 2014).

\section{RESULTS}

We recorded a total of 111 bird species belonging to 37 families and 15 orders (Tab. I) and the prevailing order was Passeriformes with 69 species. However, the quantitative survey only recorded 89 species, and the associated rarefaction curve does not reach an asymptote, indicating that several species remain undetected (Fig. 1). The IPA by species (Tab. II) ranged between 0.9375 (75 contacts) 
Tab. I. List of taxa recorded in the Mata do Cedro, state of Alagoas, Brazil in the study period through qualitative and quantitative sampling with Habitat dependency category - Forest independent (FI), Semi-dependent (SD), Forest dependent (FD); Trophic Category - aquatic (AQ), Scavenger (SC), Frugivorous (FR), Granivores (GR), Insectivorous (IN), Nectarivorous (NC), Omnivores (ON), Predators (PD); Strata - Soil (SL), Intermediate (IN), Superior (SP); Sensitivity to human disturbances - High (H), Average (AV) and Low (L); Threat category and Endemism: Vulnerable (VU), Endangered (EN), Endemic to Pernambuco Center (PC) and Endemic to Atlantic Forest (AT).

\begin{tabular}{|c|c|c|c|c|c|}
\hline Taxa & Hab. & Trop. Cat. & Strata & Sens. & Thr/End \\
\hline \multicolumn{6}{|l|}{ TINAMIFORMES } \\
\hline \multicolumn{6}{|l|}{ Tinamidae } \\
\hline Crypturellus parvirostris (Wagler, 1827) & FI & FR & $\mathrm{SL}$ & $\mathrm{L}$ & - \\
\hline Rhynchotus rufescens (Temminck, 1815) & FI & FR & SL & $\mathrm{L}$ & - \\
\hline \multicolumn{6}{|l|}{ PELECANIFORMES } \\
\hline \multicolumn{6}{|l|}{ Ardeidae } \\
\hline Butorides striata (Linnaeus, 1758) & FI & $\mathrm{AQ}$ & SL & $\mathrm{L}$ & - \\
\hline \multicolumn{6}{|l|}{ CATHARTIFORMES } \\
\hline \multicolumn{6}{|l|}{ Cathartidae } \\
\hline Cathartes aura (Linnaeus, 1758) & FI & $\mathrm{SC}$ & SP & $\mathrm{L}$ & - \\
\hline Cathartes burrovianus Cassin, 1845 & FI & $\mathrm{SC}$ & SP & A & - \\
\hline Coragyps atratus (Bechstein, 1793) & FI & $\mathrm{SC}$ & SP & $\mathrm{L}$ & - \\
\hline \multicolumn{6}{|l|}{ ACCIPITRIFORMES } \\
\hline \multicolumn{6}{|l|}{ Accipitridae } \\
\hline Leptodon forbesi (Swann, 1922) & FD & $\mathrm{PD}$ & SP & $\mathrm{A}$ & $\mathrm{EN}$ \\
\hline Geranospiza caerulescens (Vieillot, 1817) & SD & $\mathrm{PD}$ & SP & A & - \\
\hline Rupornis magnirostris (Gmelin, 1788) & FI & PD & SP & $\mathrm{L}$ & - \\
\hline Geranoaetus albicaudatus (Vieillot, 1816) & FI & $\mathrm{PD}$ & SP & $\mathrm{L}$ & - \\
\hline Buteo brachyurus Vieillot, 1816 & SD & PD & SP & A & - \\
\hline Buteo albonotatus Kaup, 1847 & FD & PD & SP & A & - \\
\hline \multicolumn{6}{|l|}{ CHARADRIIFORMES } \\
\hline \multicolumn{6}{|l|}{ Charadriidae } \\
\hline Vanellus chilensis (Molina, 1782) & FI & $\mathrm{AQ}$ & SL & $\mathrm{L}$ & - \\
\hline \multicolumn{6}{|l|}{ COLUMBIFORMES } \\
\hline \multicolumn{6}{|l|}{ Columbidae } \\
\hline Columbina talpacoti (Temminck, 1811) & FI & GR & SL & $\mathrm{L}$ & - \\
\hline Patagioenas speciosa (Gmelin, 1789) & FD & FR & SP & A & - \\
\hline Leptotila rufaxilla (Richard \& Bernard, 1792) & FD & FR & IN & A & - \\
\hline Geotrygon montana (Linnaeus, 1758) & FD & FR & SL & A & - \\
\hline \multicolumn{6}{|l|}{ CUCULIFORMES } \\
\hline \multicolumn{6}{|l|}{ Cuculidade } \\
\hline Piaya cayana (Linnaeus, 1766) & SD & $\mathrm{IN}$ & SP & $\mathrm{L}$ & - \\
\hline Crotophaga ani Linnaeus, 1758 & FI & IN & IN & $\mathrm{L}$ & - \\
\hline Tapera naevia (Linnaeus, 1766) & FI & IN & IN & $\mathrm{L}$ & - \\
\hline \multicolumn{6}{|l|}{ CAPRIMULGIFORMES } \\
\hline \multicolumn{6}{|l|}{ Caprimulgidae } \\
\hline Nyctidromus albicollis (Gmelin, 1789) & SD & IN & SL & $\mathrm{L}$ & - \\
\hline \multicolumn{6}{|l|}{ APODIFORMES } \\
\hline \multicolumn{6}{|l|}{ Trochilidae } \\
\hline Glaucis hirsutus (Gmelin, 1788) & FD & $\mathrm{NC}$ & IN & $\mathrm{L}$ & - \\
\hline Phaethornis ruber (Linnaeus, 1758) & FD & $\mathrm{NC}$ & IN & A & - \\
\hline Phaethornis pretrei (Lesson \& Delattre, 1839) & SD & $\mathrm{NC}$ & IN & $\mathrm{L}$ & - \\
\hline Anthracothorax nigricollis (Vieillot, 1817) & SD & $\mathrm{NC}$ & SP & $\mathrm{L}$ & - \\
\hline Chrysolampis mosquitus (Linnaeus, 1758) & SD & $\mathrm{NC}$ & IN & $\mathrm{L}$ & - \\
\hline Chlorestes notata (Reich, 1793) & FD & $\mathrm{NC}$ & IN & $\mathrm{L}$ & - \\
\hline Thalurania watertonii (Bourcier, 1847) & FD & $\mathrm{NC}$ & IN & A & $\mathrm{EN}, \mathrm{AT}$ \\
\hline \multicolumn{6}{|l|}{ CORACIIFORMES } \\
\hline Alcedinidae & & & & & \\
\hline Chloroceryle amazona (Latham, 1790) & SD & $\mathrm{AQ}$ & IN & $\mathrm{L}$ & - \\
\hline Chloroceryle americana (Gmelin, 1788) & SD & $\mathrm{AQ}$ & IN & $\mathrm{L}$ & - \\
\hline Momotidae & & & & & \\
\hline Momotus momota marcgrviana Pinto \& Camargo, 1961 & FD & $\mathrm{IN}$ & SP & A & $\mathrm{EN}, \mathrm{PC}$ \\
\hline GALBULIFORMES & & & & & \\
\hline Galbulidae & & & & & \\
\hline
\end{tabular}


Tab. I. Cont

\begin{tabular}{|c|c|c|c|c|c|}
\hline Taxa & Hab. & Trop. Cat. & Strata & Sens. & Thr/End \\
\hline Galbula ruficauda Cuvier, 1816 & FD & IN & IN & $\mathrm{L}$ & - \\
\hline \multicolumn{6}{|l|}{ PICIFORMES } \\
\hline \multicolumn{6}{|l|}{ Ramphastidae } \\
\hline Pteroglossus aracari (Linnaeus, 1758) & FD & FR & SP & A & - \\
\hline \multicolumn{6}{|l|}{ Picidae } \\
\hline Picumnus pernambucensis Zimmer, 1947 & FD & IN & IN & A & $\mathrm{PC}$ \\
\hline Veniliornis affinis (Swainson, 1821) & FD & IN & IN & A & - \\
\hline Veniliornis passerinus (Linnaeus, 1766) & SD & IN & IN & $\mathrm{L}$ & - \\
\hline \multicolumn{6}{|l|}{ FALCONIFORMES } \\
\hline \multicolumn{6}{|l|}{ Falconidae } \\
\hline Caracara plancus (Miller, 1777) & FI & PD & SL & $\mathrm{L}$ & - \\
\hline Milvago chimachima (Vieillot, 1816) & FI & $\mathrm{PD}$ & SP & $\mathrm{L}$ & - \\
\hline Herpetotheres cachinnans (Linnaeus, 1758) & SD & $\mathrm{PD}$ & SP & $\mathrm{L}$ & - \\
\hline Micrastur ruficollis (Vieillot, 1817) & FD & PD & IN & A & - \\
\hline \multicolumn{6}{|l|}{ PSITTACIFORMES } \\
\hline \multicolumn{6}{|l|}{ Psittacidae } \\
\hline Diopsittaca nobilis (Linnaeus, 1758) & $\mathrm{SD}$ & FR & SP & A & - \\
\hline \multicolumn{6}{|l|}{ PASSERIFORMES } \\
\hline \multicolumn{6}{|l|}{ Thamnophilidae } \\
\hline Myrmotherula axillaris (Vieillot, 1817) & FD & IN & IN & A & - \\
\hline Formicivora grisea (Boddaert, 1783) & SD & IN & IN & $\mathrm{L}$ & - \\
\hline Dysithamnus mentalis (Temminck, 1823) & FD & IN & IN & A & - \\
\hline Herpsilochmus rufimarginatus (Temmick, 1822) & FD & IN & SP & A & - \\
\hline Thamnophilus aethiops distans Pinto, 1954 & FD & IN & SL & $\mathrm{H}$ & $\mathrm{EN}, \mathrm{PC}$ \\
\hline Myrmoderus ruficauda soror Pinto, 1940 & FD & IN & SL & A & $\mathrm{EN}, \mathrm{PC}$ \\
\hline Pyriglena pernambucensis Zimmer, 1931 & FD & IN & SL & A & VU, PC \\
\hline \multicolumn{6}{|l|}{ Conopophagidae } \\
\hline Conopophaga melanops nigrifrons Pinto, 1954 & FD & IN & IN & $\mathrm{H}$ & VU, PC \\
\hline \multicolumn{6}{|l|}{ Formicariidae } \\
\hline Formicarius colma Boddaert, 1783 & FD & $\mathrm{IN}$ & SL & $\mathrm{H}$ & - \\
\hline \multicolumn{6}{|l|}{ Dendrocolaptidae } \\
\hline Dendrocincla taunayi Pinto, 1939 & FD & IN & IN & $\mathrm{H}$ & $\mathrm{EN}, \mathrm{PC}$ \\
\hline Sittasomus griseicapillus (Vieillot, 1818) & FD & IN & IN & A & - \\
\hline Xiphorhynchus guttatus (Lichtenstein, 1820) & FD & IN & IN & A & - \\
\hline Dendroplex picus (Gmelin, 1788) & SD & IN & IN & $\mathrm{L}$ & - \\
\hline \multicolumn{6}{|l|}{ Xenopidae } \\
\hline Xenops minutus alagoanus Pinto, 1954 & FD & IN & IN & A & VU, PC \\
\hline \multicolumn{6}{|l|}{ Furnariidae } \\
\hline Synallaxis frontalis Pelzeln, 1859 & SD & IN & IN & $\mathrm{L}$ & - \\
\hline \multicolumn{6}{|l|}{ Pipridae } \\
\hline Neopelma pallescens (Lafresnaye, 1853) & FD & FR & IN & A & - \\
\hline Ceratopipra rubrocapilla (Temminck, 1821) & FD & FR & SP & $\mathrm{H}$ & - \\
\hline Manacus manacus (Linnaeus, 1766) & FD & FR & IN & $\mathrm{L}$ & - \\
\hline Chiroxiphia pareola (Linnaeus, 1766) & FD & FR & IN & $\mathrm{H}$ & - \\
\hline \multicolumn{6}{|l|}{ Tityridae } \\
\hline Schiffornis turdina intermedia Pinto, 1954 & FD & FR & IN & $\mathrm{H}$ & VU, PC \\
\hline \multicolumn{6}{|l|}{ Platyrinchidae } \\
\hline Platyrinchus mystaceus niveigularis Pinto, 1954 & FD & IN & IN & $\mathrm{H}$ & VU, PC \\
\hline Rhynchocyclidae & & & & & \\
\hline Mionectes oleagineus (Lichtenstein, 1823) & SD & FR & IN & A & - \\
\hline Leptopogon amaurocephalus Tschudi, 1846 & FD & FR & IN & A & - \\
\hline Tolmomyias poliocephalus (Taczanowski, 1884) & FD & IN & SP & A & - \\
\hline Tolmomyias flaviventris (Wied, 1831) & FD & IN & SP & $\mathrm{L}$ & - \\
\hline Todirostrum cinereum (Linnaeus, 1766) & SD & IN & $\mathrm{IN}$ & $\mathrm{L}$ & - \\
\hline Hemitriccus griseipectus naumburgae (Zimmer, 1945) & FD & IN & IN & $\mathrm{H}$ & VU, PC \\
\hline Tyrannnidae & & & & & \\
\hline Ornithion inerme Hartlaub, 1853 & FD & $\mathrm{IN}$ & SP & A & - \\
\hline Camptostoma obsoletum (Temminck, 1824) & FI & IN & SP & $\mathrm{L}$ & - \\
\hline
\end{tabular}


Tab. I. Cont

\begin{tabular}{|c|c|c|c|c|c|}
\hline Taxa & Hab. & Trop. Cat. & Strata & Sens. & Thr/End \\
\hline Elaenia flavogaster (Thunberg, 1822) & SD & FR & SP & $\mathrm{L}$ & - \\
\hline Myiopagis gaimardii (d’Orbigny, 1839) & FD & FR & SP & A & - \\
\hline Myiopagis caniceps (Swainson, 1835) & FD & FR & SP & A & - \\
\hline Capsiempis flaveola (Lichtenstein, 1823) & SD & IN & IN & $\mathrm{L}$ & - \\
\hline Attila spadiceus (Gmelin, 1789) & FD & IN & SP & A & - \\
\hline Legatus leucophaius (Vieillot, 1818) & FD & FR & IN & $\mathrm{L}$ & - \\
\hline Myiarchus ferox (Gmelin, 1789) & SD & IN & IN & $\mathrm{L}$ & - \\
\hline Rhytipterna simplex (Lichtenstein, 1823) & FD & IN & IN & $\mathrm{H}$ & - \\
\hline Pitangus sulphuratus (Linnaeus, 1766) & FI & IN & SP & $\mathrm{L}$ & - \\
\hline Megarynchus pitangua (Linnaeus, 1766) & FD & IN & SP & $\mathrm{L}$ & - \\
\hline Myiozetetes similis (Spix, 1825) & $\mathrm{SD}$ & IN & SP & $\mathrm{L}$ & - \\
\hline Tyrannus melancholicus Vieillot, 1819 & FI & IN & SP & $\mathrm{L}$ & - \\
\hline Arundinicola leucocephala (Linnaeus, 1764) & FI & IN & IN & A & - \\
\hline Lathrotriccus euleri (Cabanis, 1868) & FD & IN & IN & A & - \\
\hline \multicolumn{6}{|l|}{ Vireonidae } \\
\hline Cyclarhis gujanensis (Gmelin, 1789) & FD & IN & IN & $\mathrm{L}$ & - \\
\hline Vireo chivi (Vieillot, 1817) & FD & IN & IN & $\mathrm{L}$ & - \\
\hline \multicolumn{6}{|l|}{ Hirundinidae } \\
\hline Stelgidopteryx ruficollis (Vieillot, 1817) & FI & IN & SP & $\mathrm{L}$ & - \\
\hline Progne tapera (Vieillot, 1817) & FI & IN & SP & $\mathrm{L}$ & - \\
\hline Tachycineta albiventer (Boddaert, 1783) & FI & IN & IN & A & - \\
\hline Hirundo rustica Linnaeus, 1758 & FI & IN & SP & $\mathrm{L}$ & - \\
\hline \multicolumn{6}{|l|}{ Troglodytidae } \\
\hline Troglodytes musculus Naumann, 1823 & FI & IN & IN & $\mathrm{L}$ & - \\
\hline Pheugopedius genibarbis (Swainson, 1838) & FD & $\mathrm{ON}$ & IN & $\mathrm{L}$ & - \\
\hline \multicolumn{6}{|l|}{ Donacobiidae } \\
\hline Donacobius atricapilla (Linnaeus, 1766) & FI & IN & IN & A & - \\
\hline \multicolumn{6}{|l|}{ Turdidae } \\
\hline Turdus leucomelas Vieillot, 1818 & $\mathrm{SD}$ & $\mathrm{ON}$ & IN & $\mathrm{L}$ & - \\
\hline Turdus fumigatus Lichtenstein, 1823 & FD & ON & IN & A & - \\
\hline \multicolumn{6}{|l|}{ Passerellidae } \\
\hline Ammodramus humeralis (Bosc, 1792) & FI & GR & SL & $\mathrm{L}$ & - \\
\hline Arremon taciturnus (Hermann, 1783) & FD & ON & SL & $\mathrm{L}$ & - \\
\hline \multicolumn{6}{|l|}{ Parulidae } \\
\hline Basileuterus culicivorus (Deppe, 1830) & FD & IN & IN & A & - \\
\hline \multicolumn{6}{|l|}{ Thraupidae } \\
\hline Tangara palmarum (Wied, 1823) & SD & FR & SP & $\mathrm{L}$ & - \\
\hline Nemosia pileata (Boddaert, 1783) & SD & FR & SP & $\mathrm{L}$ & - \\
\hline Hemithraupis guira (Linnaeus, 1766) & FD & FR & SP & $\mathrm{L}$ & - \\
\hline Volatinia jacarina (Linnaeus, 1766) & FI & GR & IN & $\mathrm{L}$ & - \\
\hline Lanio cristatus (Linnaeus, 1766) & FD & FR & SP & A & - \\
\hline Dacnis cayana (Linnaeus, 1766) & FD & FR & SP & $\mathrm{L}$ & - \\
\hline Coereba flaveola (Linnaeus, 1758) & SD & $\mathrm{NC}$ & SP & $\mathrm{L}$ & - \\
\hline Saltator maximus (Statius Muller, 1776) & FD & ON & IN & $\mathrm{L}$ & - \\
\hline \multicolumn{6}{|l|}{ Fringillidae } \\
\hline Euphonia chlorotica (Linnaeus, 1766) & SD & FR & SP & $\mathrm{L}$ & - \\
\hline Euphonia violacea (Linnaeus, 1758) & FD & FR & SP & $\mathrm{L}$ & - \\
\hline \multicolumn{6}{|l|}{ Estrildidae } \\
\hline Estrilda astrild (Linnaeus, 1758) & FI & GR & IN & $\mathrm{L}$ & - \\
\hline
\end{tabular}

for Blue-backed Manakin Chiroxiphia pareola and 0.0125 (one contact) in 16 species whereas the value of the diversity index was 3.87 and the evenness index was 0.86 .

The frequency of occurrence of the 89 species recorded in the quantitative survey revealed that 12 species had $100 \%$ FO and 14 had $12.5 \%$ FO (one contact). The category of abundance (status) of these species consisted of $66 \%$ resident species, $16 \%$ wandering, $13 \%$ occasional and $4 \%$ migrating species (Tab. II). Forest dependent species were the most represented, making up $51.35 \%$ of records $(n=57)$. Semi-dependent $(\mathrm{n}=27)$ and independent $(\mathrm{n}=27)$ species corresponded to the same percentage (24.32\%) (Tab. I). 
Tab. II. Spot Index of Abundance (IPA) found for each species based on count points, frequency of occurrence (FO) and status: resident (RE), occasional (OC), wandering (WA) and migration (MI) in the Mata do Cedro, state of Alagoas, Brazil.

\begin{tabular}{|c|c|c|c|c|}
\hline Taxa & Total of contacts & IPA & $\mathrm{FO} \%$ & Status \\
\hline Crypturellus parvirostris (Wagler, 1827) & 3 & 0.04 & 37.50 & $\mathrm{OC}$ \\
\hline Butorides striata (Linnaeus, 1758) & 1 & 0.01 & 25.00 & $\mathrm{OC}$ \\
\hline Cathartes aura (Linnaeus, 1758) & 2 & 0.03 & 50.00 & $\mathrm{OC}$ \\
\hline Cathartes burrovianus Cassin, 1845 & 3 & 0.04 & 37.50 & $\mathrm{OC}$ \\
\hline Coragyps atratus (Bechstein, 1793) & 3 & 0.04 & 25.00 & $\mathrm{OC}$ \\
\hline Leptodon forbesi (Swann, 1922) & 2 & 0.03 & 12.50 & WA \\
\hline Geranospiza caerulescens (Vieillot, 1817) & 1 & 0.01 & 12.50 & WA \\
\hline Rupornis magnirostris (Gmelin, 1788) & 2 & 0.03 & 25.00 & $\mathrm{OC}$ \\
\hline Caracara plancus (Miller, 1777) & 5 & 0.06 & 25.00 & $\mathrm{OC}$ \\
\hline Herpetotheres cachinnans (Linnaeus, 1758) & 1 & 0.01 & 12.50 & WA \\
\hline Micrastur ruficollis (Vieillot, 1817) & 3 & 0.04 & 37.50 & $\mathrm{RE}$ \\
\hline Vanellus chilensis (Molina, 1782) & 5 & 0.06 & 37.50 & $\mathrm{OC}$ \\
\hline Patagioenas speciosa (Gmelin, 1789) & 13 & 0.16 & 100.00 & $\mathrm{RE}$ \\
\hline Leptotila verreauxi Bonaparte, 1855 & 1 & 0.01 & 12.50 & WA \\
\hline Leptotila rufaxilla (Richard \& Bernard, 1792) & 1 & 0.01 & 12.50 & WA \\
\hline Geotrygon montana (Linnaeus, 1758) & 2 & 0.03 & 37.50 & $\mathrm{RE}$ \\
\hline Diopsittaca nobilis (Linnaeus, 1758) & 8 & 0.10 & 87.50 & $\mathrm{RE}$ \\
\hline Piaya cayana (Linnaeus, 1766) & 10 & 0.13 & 75.00 & $\mathrm{RE}$ \\
\hline Crotophaga ani Linnaeus, 1758 & 2 & 0.03 & 37.50 & $\mathrm{OC}$ \\
\hline Tapera naevia (Linnaeus, 1766) & 1 & 0.01 & 12.50 & WA \\
\hline Glaucis hirsutus (Gmelin, 1788) & 1 & 0.01 & 12.50 & $\mathrm{~V}$ \\
\hline Phaethornis ruber (Linnaeus, 1758) & 9 & 0.11 & 75.00 & $\mathrm{RE}$ \\
\hline Phaethornis pretrei (Lesson \& Delattre, 1839) & 1 & 0.01 & 12.50 & WA \\
\hline Chlorostilbon notatus (Reich, 1793) & 2 & 0.03 & 25.00 & $\mathrm{RE}$ \\
\hline Thalurania watertonii (Bourcier, 1847) & 1 & 0.01 & 12.50 & WA \\
\hline Chloroceryle amazona (Latham, 1790) & 1 & 0.01 & 12.50 & WA \\
\hline Galbula ruficauda Cuvier, 1816 & 5 & 0.06 & 62.50 & $\mathrm{RE}$ \\
\hline Pteroglossus aracari (Linnaeus, 1758) & 1 & 0.01 & 12.50 & WA \\
\hline Picumnus pernambucensis Zimmer, 1947 & 13 & 0.16 & 75.00 & $\mathrm{RE}$ \\
\hline Veniliornis affinis (Swainson, 1821) & 2 & 0.03 & 25.00 & $\mathrm{RE}$ \\
\hline Veniliornis passerinus (Linnaeus, 1766) & 2 & 0.03 & 25.00 & $\mathrm{RE}$ \\
\hline Myrmoderus ruficauda soror Pinto, 1940 & 1 & 0.01 & 25.00 & $\mathrm{RE}$ \\
\hline Myrmotherula axillaris (Vieillot, 1817) & 16 & 0.20 & 100.00 & $\mathrm{RE}$ \\
\hline Formicivora grisea (Boddaert, 1783) & 8 & 0.10 & 100.00 & $\mathrm{RE}$ \\
\hline Dysithamnus mentalis (Temminck, 1823) & 13 & 0.16 & 87.50 & $\mathrm{RE}$ \\
\hline Herpsilochmus rufimarginatus (Temminck, 1822) & 26 & 0.33 & 100.00 & $\mathrm{RE}$ \\
\hline Thamnophilus aethiops distans Pinto, 1954 & 13 & 0.16 & 87.50 & $\mathrm{RE}$ \\
\hline Pyriglena pernambucensis Zimmer, 1931 & 12 & 0.15 & 87.50 & $\mathrm{RE}$ \\
\hline Conopophaga melanops nigrifrons Pinto, 1943 & 12 & 0.15 & 100.00 & $\mathrm{RE}$ \\
\hline Formicarius colma Boddaert, 1783 & 6 & 0.08 & 75.00 & $\mathrm{RE}$ \\
\hline Dendrocincla taunayi Pinto, 1939 & 4 & 0.05 & 25.00 & $\mathrm{RE}$ \\
\hline Xiphorhynchus guttatus (Lichtenstein, 1820) & 26 & 0.33 & 12.50 & WA \\
\hline Dendroplex picus (Gmelin, 1788) & 1 & 0.01 & 100.00 & $\mathrm{RE}$ \\
\hline Xenops minutus alagoanus Pinto, 1954 & 10 & 0.13 & 25.00 & $\mathrm{RE}$ \\
\hline Neopelma pallescens (Lafresnaye, 1853) & 6 & 0.08 & 87.50 & $\mathrm{RE}$ \\
\hline Ceratopipra rubrocapilla Temminck, 1821 & 47 & 0.59 & 62.50 & $\mathrm{RE}$ \\
\hline Manacus manacus (Linnaeus, 1766) & 10 & 0.13 & 100.00 & $\mathrm{RE}$ \\
\hline Chiroxiphia pareola (Linnaeus, 1766) & 75 & 0.94 & 62.50 & $\mathrm{RE}$ \\
\hline Schiffornis turdina intermedia Pinto, 1954 & 19 & 0.24 & 100.00 & $\mathrm{RE}$ \\
\hline Platyrinchus mystaceus niveigularis Pinto, 1954 & 15 & 0.19 & 100.00 & $\mathrm{RE}$ \\
\hline Mionectes oleagineus (Lichtenstein, 1823) & 3 & 0.04 & 75.00 & $\mathrm{RE}$ \\
\hline Leptopogon amaurocephalus Tschudi, 1846 & 5 & 0.06 & 50.00 & $\mathrm{RE}$ \\
\hline Tolmomyias poliocephalus (Taczanowski, 1884) & 13 & 0.16 & 62.50 & $\mathrm{RE}$ \\
\hline Tolmomyias flaviventris (Wied, 1831) & 53 & 0.66 & 75.00 & $\mathrm{RE}$ \\
\hline Hemitriccus griseipectus naumburgae (Zimmer, 1945) & 28 & 0.35 & 100.00 & $\mathrm{RE}$ \\
\hline Ornithion inerme Hartlaub, 1853 & 3 & 0.04 & 100.00 & $\mathrm{RE}$ \\
\hline Elaenia flavogaster (Thunberg, 1822) & 4 & 0.05 & 37.50 & $\mathrm{RE}$ \\
\hline
\end{tabular}


Tab. II. Cont.

\begin{tabular}{|c|c|c|c|c|}
\hline Taxa & Total of contacts & IPA & $\mathrm{FO} \%$ & Status \\
\hline Myiopagis gaimardii (d’Orbigny, 1839) & 7 & 0.09 & 62.50 & $\mathrm{RE}$ \\
\hline Myiopagis caniceps (Swainson, 1835) & 4 & 0.05 & 62.50 & $\mathrm{RE}$ \\
\hline Attila spadiceus (Gmelin, 1789) & 8 & 0.10 & 37.50 & $\mathrm{RE}$ \\
\hline Legatus leucophaius (Vieillot, 1818) & 6 & 0.08 & 75.00 & $\mathrm{RE}$ \\
\hline Myiarchus ferox (Gmelin, 1789) & 1 & 0.01 & 37.50 & $\mathrm{RE}$ \\
\hline Rhytipterna simplex (Lichtenstein, 1823) & 16 & 0.20 & 25.00 & $\mathrm{RE}$ \\
\hline Pitangus sulphuratus (Linnaeus, 1766) & 8 & 0.10 & 87.50 & $\mathrm{RE}$ \\
\hline Megarynchus pitangua (Linnaeus, 1766) & 6 & 0.08 & 87.50 & $\mathrm{RE}$ \\
\hline Myiozetetes similis (Spix, 1825) & 10 & 0.13 & 62.50 & $\mathrm{RE}$ \\
\hline Tyrannus melancholicus Vieillot, 1819 & 4 & 0.05 & 87.50 & $\mathrm{RE}$ \\
\hline Lathrotriccus euleri (Cabanis, 1868) & 2 & 0.03 & 75.00 & $\mathrm{RE}$ \\
\hline Cyclarhis gujanensis (Gmelin, 1789) & 2 & 0.03 & 25.00 & $\mathrm{RE}$ \\
\hline Vireo chivi (Vieillot, 1817) & 30 & 0.38 & 50.00 & $\mathrm{OC}$ \\
\hline Stelgidopteryx ruficollis (Vieillot, 1817) & 4 & 0.05 & 75.00 & MI \\
\hline Progne tapera (Vieillot, 1817) & 7 & 0.09 & 50.00 & MI \\
\hline Tachycineta albiventer (Boddaert, 1783) & 3 & 0.04 & 37.50 & MI \\
\hline Hirundo rustica Linnaeus, 1758 & 1 & 0.01 & 75.00 & $\mathrm{OC}$ \\
\hline Pheugopedius genibarbis (Swainson, 1838) & 47 & 0.59 & 25.00 & MI \\
\hline Coereba flaveola (Linnaeus, 1758) & 13 & 0.16 & 100.00 & $\mathrm{RE}$ \\
\hline Saltator maximus (Statius Muller, 1776) & 12 & 0.15 & 75.00 & $\mathrm{RE}$ \\
\hline Nemosia pileata (Boddaert, 1783) & 3 & 0.04 & 87.50 & $\mathrm{RE}$ \\
\hline Lanio cristatus (Linnaeus, 1766) & 8 & 0.10 & 50.00 & $\mathrm{RE}$ \\
\hline Tangara palmarum (Wied, 1823) & 7 & 0.09 & 87.50 & $\mathrm{RE}$ \\
\hline Dacnis cayana (Linnaeus, 1766) & 7 & 0.09 & 75.00 & $\mathrm{RE}$ \\
\hline Hemithraupis guira (Linnaeus, 1766) & 3 & 0.04 & 75.00 & $\mathrm{RE}$ \\
\hline Ammodramus humeralis (Bosc, 1792) & 2 & 0.03 & 12.50 & WA \\
\hline Volatinia jacarina (Linnaeus, 1766) & 2 & 0.03 & 12.50 & WA \\
\hline Arremon taciturnus (Hermann, 1783) & 3 & 0.04 & 37.50 & $\mathrm{RE}$ \\
\hline Basileuterus culicivorus (Deppe, 1830) & 5 & 0.06 & 62.50 & $\mathrm{RE}$ \\
\hline Euphonia chlorotica (Linnaeus, 1766) & 1 & 0.01 & 25.00 & $\mathrm{OC}$ \\
\hline Euphonia violacea (Linnaeus, 1758) & 11 & 0.14 & 75.00 & $\mathrm{RE}$ \\
\hline
\end{tabular}

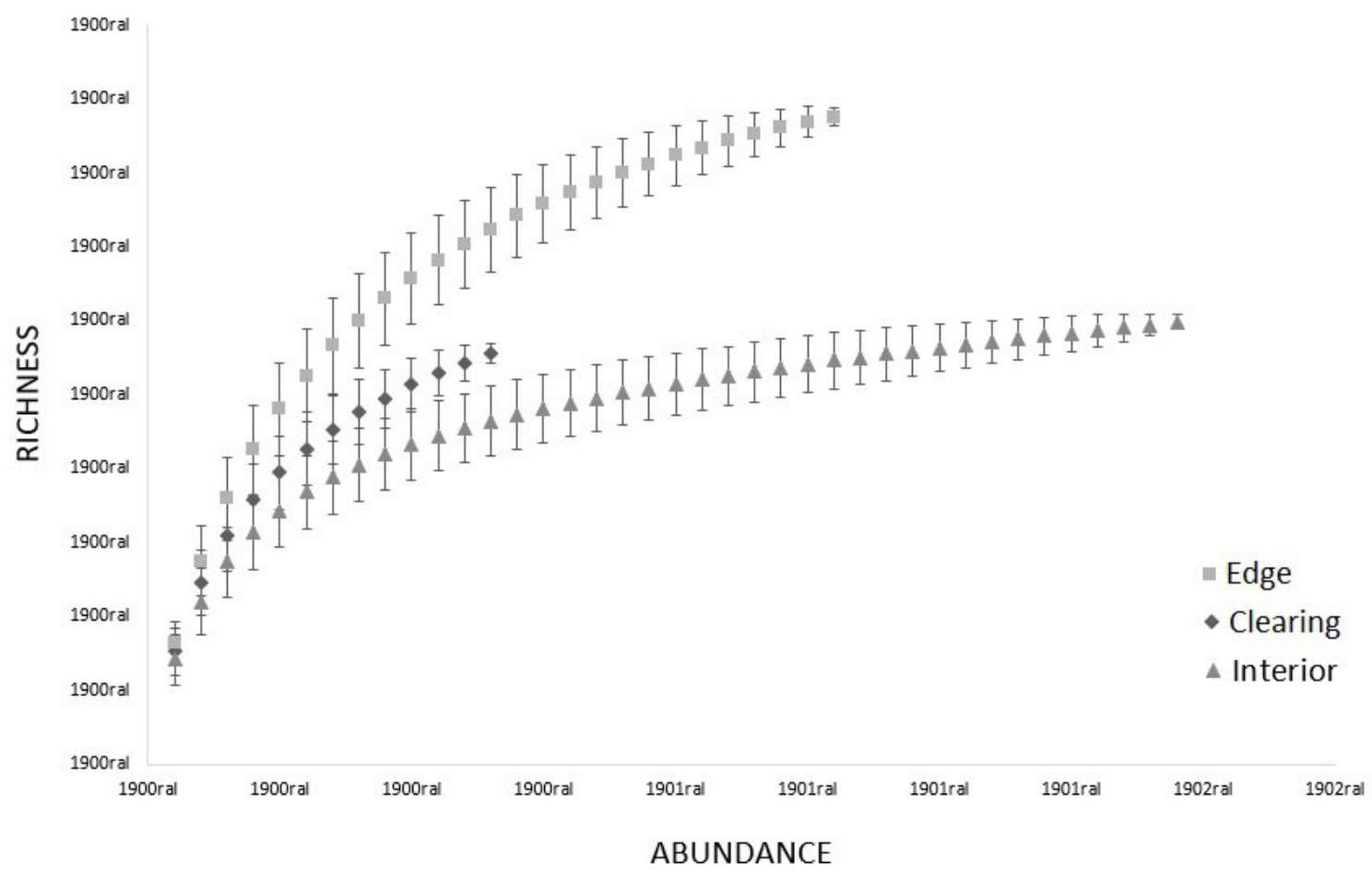

Fig. 1. Rarefaction curve based at Mata do Cedro abundance and richness, state of Alagoas, Brazil. 
Insectivores $(46 \% \mathrm{n}=51)$ and frugivorous $(23 \% \mathrm{n}=26)$ were the most significant trophic categories with other categories accounting for $31 \%$ of species.

Intermediate and superior strata were most used, with $80 \%$ of the species occupying these two strata. The soil stratum was occupied by 14 species (13\%) with eight species (7\%) using the vertical stratum. Of all species registered, $11(10 \%)$ showed high, $39(35 \%)$ had average and $61(55 \%)$ had low sensitivity to human disturbance. Twelve species are classified as threated and 11 are considered endemic to the PCE and the Atlantic Forest.

The dendrogram separated one species (the Common Waxbill Estrilda astrild Linnaeus, 1758) by its grazing habit; and grouped the others according to their different diets and foraging habits (Fig. 2).

\section{DISCUSSION}

The bird community of Mata de Cedro is highly diverse, with the presence of endemic and threatened taxa, balanced trophic categories: characteristics of communities in well preserved tropical forests. Our results therefore reinforce the importance of Mata do Cedro for the maintenance of local biodiversity and highlight their probable role as stepping stones for birds moving through this highly fragmented region. Previous studies in Mata do Cedro identified 45 forest dependent species, 11 PCE endemics and 12 threatened taxa (PEREIRA et al. 2016), strongly corroborating the results of the present work.

Compared to previous studies in the PCE, the present study found higher species richness than LYRA-NEVES et al. (2004), and Lobo-Araújo et al. (2013), probably due to particularities of different forest types, the small sizes of the fragments or differences in sampling effort and methodologies. Conversely, RoDA (2004), MagalHãES et al. (2007) and FARIAS et al. (2007) recorded higher values of 125,151 and 140 species, respectively, in contrast to the 111 found in this study. In Alagoas, DAHER et al. (2013) registered 150 and 110 species in Madeiras and Fazenda Brejo, respectively. The application of long term studies and the adoption of different sampling methods may further improve our knowledge of species richness in highly diverse areas such as the Atlantic Forest (MoRAEs et al., 2007).

The observed abundance patterns are consistent with the literature, with few species showing high values and large number of species showing intermediate and low values (DONATELLI et al., 2004; LYRA-NEVES et al., 2004; DARIO, 2010). These results are typical of bird communities in tropical forests, with high species richness and low number of individuals in each species, except for some naturally abundant taxa (Donatelli et al., 2004). Likewise, the diversity and evenness indices indicate that most species are equally abundant in the environment (MAGURRAN, 1988), supporting similar findings by VIELLIARD \& SiLva (1990) $\left(\mathrm{H}^{\prime}=3.89\right.$ and $\mathrm{E}=0.83$ for a forest fragment of $\left.592 \mathrm{ha}\right)$ - considered normal for effectively sampled neotropical forest environments. Indeed, the larger the forest fragment, the greater its diversity indices due to the complexity of the environment and availability of niches (RoDA, 2004). In fragments smaller than 100 ha, diversity is reduced dramatically due to edge effects and the strong inflow of generalist species.

Several resident and forest dependent species were registered in Mata do Cedro. However, most were classified as wandering, occasional or migratory species. In addition, we recorded several species that are typically found in open areas such as fields and wetlands (24\%), demonstrating the importance of this fragment for reducing isolation and therefore increasing persistence across wider spatial and temporal scales (SAURA et al., 2014).

Forest fragments are both directly and indirectly affected by their isolation, size and shape, type of surrounding matrix and edge effect (BIERREGAARD et al., 1992; RANTA et al., 1998). As a consequence, birds populations can be reduced or become locally extinct; processes that particularly effect insectivorous understory taxa, large frugivores and raptors (AlEIXO \& VIELLIARD, 1995; OFFERMAN et al., 1995; Jullien \& THIOLLAY, 1996). In 2002, SilveIRA et al. (2003) recorded 78 species for the Mata do Cedro, 15 of which were not sampled in this study. Among these is the Bearded Bellbird Procnias averano (Hermann, 1783) and Lettered Aracari Pteroglossus inscriptus Swainson, 1822, both large frugivores. Their absence may be evidence of local extinction caused by pressures in the area such as hunting, captivity and other effects discussed above.

A total of 10 recorded species were classified as highly sensitive to human disturbance. Although this number is lower than that found by LYRA-NEves et al. (2004) (16) and Rodrigues et al. (2007) (17) in other areas of the PCE, the presence of these species indicate good conservation conditions (RoDA, 2004) of the Mata do Cedro. The low abundance (mean IPA $=0.3 \pm 0.3$ ) of these species may be related to a greater sensitivity to forest fragmentation (ANJOS, 2006). Besides that, such sensitivity can increase the risk of local extinction (e.g. Purvis et al., 2000) and their presence should be used to assign conservation priorities (ANJOs et al., 2011). The presence of these species also demonstrates the importance of the Mata do Cedro for local conservation of these populations. It is also important to note that even such an isolated forest fragment can provide an important refuge for sensitive species, facilitating long-distance dispersal and functioning as stepping stones to other forest patches (UEZU et al., 2008; KrAMER-SCHADT et al., 2011).

Similarly, mapping trophic relationships is important for evaluating a bird community (RoDRIGUES et al., 2007). Most species in Mata do Cedro were insectivores or frugivores. These results corroborate previous studies (PIRATELLI \& Pereira, 2002; Donatelli et al., 2004; Roda, 2004; RoDA \& PEREIRA, 2005), who claim that this distribution is characteristic of well-preserved tropical forests. Other authors, however, have shown a predominance of insectivores and omnivores in tropical bird communities (TELINO-JR et al., 2005; RoDrigues et al., 2007; DARIO, 2008). 


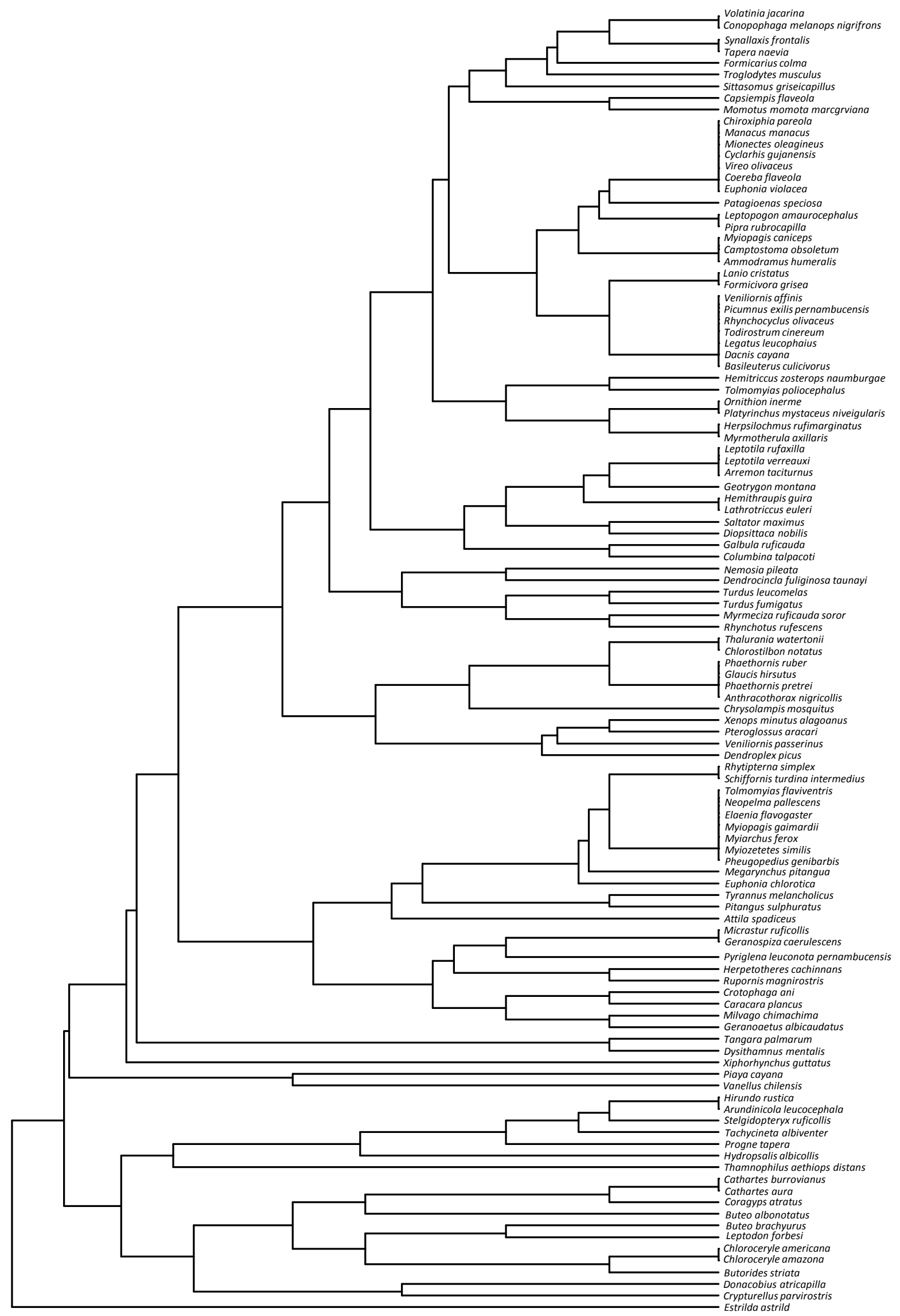

Fig. 2. Functional dendrogram of species of birds found at Mata do Cedro, state of Alagoas, Brazil. 
In this study approximately $57 \%(\mathrm{n}=29)$ of insectivores were forest dependent, particularly climbers of trunks and branches or relying on understory and soil strata. The latter comprise the forest dependent species with limited dispersal ability and so are strongly affected by fragmentation, changes in vegetation structure and loss of associated organisms, such as ants (SiLVEIRA et al., 2003). According to WiLLIS (1979) environmental changes can lead to increases in omnivorous birds and less specialized insectivores and a decrease of more specialized frugivores and insectivores.

The Bearded Bellbird Procnias averano (Hermann, 1783) seems to be locally extinct and Pteroglossus aracari (Linnaeus, 1758) was only registered with one individual. In addition, of the three recorded terrestrial frugivores, two (Small-billed Tinamou Crypturellus parvirostris (Wagler, 1827) and Red-winged Tinamou Rhynchotus rufescens (Temmick, 1815)) are forest independent and the third (the Ruddy Quail-Dove Geotrygon montana (Linnaeus, 1758)) is forest dependent and is restricted to the area and neighboring fragments. In fact, despite being able to move between fragments in search of food, large frugivores may not be able to cross the surrounding matrix (RODA, 2004). So, the reduction in habitat may be responsible for the loss of species that require large areas of forest (ALEIXo \& VIELLIARD, 1995), such as the large frugivores and large predators (S. A. Roda, dados inéditos).

We recorded 10 species of raptors, among them Leptodon forbesi, an endemic of the Atlantic Forest and severely threatened in northeast Brazil (F. V. Dénes, dados inéditos). Predators have high influence on prey distributions, since perceived risk of predation can affect prey behavior (SODHI, 1990), stabilize predator-prey dynamics (Ives \& Dobson, 1987) and lead to higher species richness via competitive coexistence (BRown et al., 1988). Furthermore, predator species have a functional role related to the recycling of carcasses and limiting the spread of diseases and undesirable mammalian scavengers (PrAKASH et al., 2003). Therefore, once again, the data obtained in this study demonstrates the importance of these species for the ecological functioning and conservation of the habitat and for the PCE in general, where the situation of birds of prey is very worrying (see RoDA \& PEREIRA, 2006).

In addition, we recorded ten species of granivores and omnivores, with six forest dependents and one (Redshouldered Macaw Diopsittaca nobilis Linaeus, 1758) with medium sensitivity to disturbances. All others were classified as having low sensitivity. Granivory plays a crucial role in the regeneration, colonization ability and spatial distribution of plants. In addition, granivores have been suggested as agents of natural selection, influencing seed trait and seed production strategies (chemical compounds, growth rate) (Hulme \& BenKman, 2002). Therefore, the dispersal of seeds by birds facilitates the regeneration of deforested and other marginal habitats (SEKERCIOGLU, 2006), and the presence of these species in the fragment is therefore important for ecosystem functioning.
Similarly, the presence of seven species of nectarivores in the study site is a positive signal for conservation. Bird species pollinate approximately 500 genera of vascular plants (SEKercioglu, 2006). In addition, to meet their high energy needs, they visit numerous flowers regularly, which increases gene flow among plants (SCHUCHMANN, 1999). Avian pollination has ecological, economic, evolutionary and conservation significance in species-rich communities such as tropical forests. Amongst the nectarivores at the study site, the Long-tailed Woodnymph Thalurania watertonii (Bourcier, 1847) is considered a vulnerable species endemic to the Atlantic forest, with medium sensitivity to disturbances. Its conservation, along with the other species responsible for this functional role, will help to maintain the local plant community and the conservation of the fragment. Recently, the hummingbird Phaethornis margarettae camargoi Grantsau, 1988 was recorded in the Mata do Cedro (G. A. Pereira, dados inéditos). This hummingbird is considered critically endangered, and there is little information about its natural history and geographical distribution (G. A. Pereira, dados inéditos).

The functional groups responsible for the consumption of insects (gleaning, gleaning on air, gleaning on soil and vegetation, probing soil and vegetation, pouncing on soil and vegetation) are extremely important for the maintenance of the fragment, due to their effects on herbivorous arthropods, since these strongly impact plants and their associated nutritional cycles (BоHм et al., 2011). In addition, herbivorous arthropods can reduce leaf area through feeding activities and hence affect the biomass of trees (BоHM et al., 2011). VAN BAEL et al. (2008) fond that foliage-gleaning birds can limit herbivorous arthropod densities and their damage to plants in tropical forests. In tropical systems, species richness of insectivorous birds is the main controlling factor for arthropods, because species richness of predators correlates with functional richness (VAN BAEL et al., 2008). Hence, higher species richness of predators increases the probability that highly efficient species are present (Рerfecto, 2004; PHILPOTT et al., 2009). At our study site, 75 insectivorous and omnivorous bird species were recorded, including species that adopted gleaning, probing and pouncing strategies. Ten of these species are threatened and all of these are restricted to the PCE and have medium and high sensitivity to disturbances and occupy various strata.

Bонм et al. (2011) showed that top-down control of leaf damage depends on predator diversity. Changes in species richness and abundance of birds through human induced changes in land use might therefore have far-reaching consequences for ecosystem functioning and services, as a decrease in species richness is likely to lead to a steep increase in the abundance of arthropod herbivores. In turn, this might influence the intensity of leaf damage and, ultimately, forest productivity. Hence, the maintenance of insectivorous birds at Mata do Cedro is crucial for its long-term conservation.

The function of predators in food webs is assumed to be positively related with species richness and abundance (BоHм et al., 2011). Habitat fragmentation causes local 
extinction and has long-term effects on populations through changes in ecological processes. As a result, fragmented ecosystems usually fail to support species assemblages found in intact ecosystems (LovEJoy et al., 1984). Due to its complex ecological structure and high species diversity, species loss through fragmentation is especially high in tropical forests (WILCOVE et al., 1986). In addition, declines in bird populations, even before they are well studied, can rapidly diminish certain ecosystem processes, such as seed dispersal, plant growth and nutrient cycling (SEKERCIOGLU, 2006). The Atlantic Forest currently exists as isolated fragments. Conservation efforts must focus on the remaining fragments, such as the Mata do Cedro, which are important in reducing regional isolation, functioning as stepping stones. (Schelhas \& GreenberG, 1996; Ribeiro et al., 2009).

\section{REFERENCES}

Aleixo, A. \& Vielliard, J. M. E. 1995. Composição e dinâmica da avifauna da Mata de Santa Genebra, Campinas, São Paulo, Brasil. Revista Brasileira de Zoologia 12:493-511.

Almeida, M. E. C.; Vielliard, J. M. E. \& Dias, M. M. 1999. Composição da avifauna em duas matas ciliares na bacia do rio Jacaré-Pepira, São Paulo. Revista Brasileira de Zoologia 16:1087-1098.

AnJos, L. 2006. Bird species sensitivity in a fragmented landscape of the Atlantic Forest Southern Brazil. Biotropica 32:229-234.

Anjos, L.; Collins, C. A.; Holt, R. D.; Volpato, G. H.; Mendonça, L. B.; Lopes, E. V.; Boçon, R.; Bisheimer, M. V.; Serafini, P. P.; Carvalho, J. 2011. Bird species abundance-occupancy patterns and sensitivity to forest fragmentation: implications for conservation in the Brazilian Atlantic Forest. Biological Conservation 144:2213-2222.

Antunes, A. Z. 2005. Alteração na composição da Comunidade de aves ao longo do tempo em um fragmento florestal no Sudeste do Brasil. Ararajuba 13:47-61.

Bierregaard, B. O.; Lovejoy, T. E.; Kapos, V.; Santos, A. A. Dos \& Hutchings, R. W. 1992. The biological dynamics of tropical rainforest Fragments. Bioscience 42:859-866.

Boнm, S. M.; Wells, K. \& KalKo, E. K. V. 2011. Top-Down control of herbivory by birds and bats in the canopy of Temperate Broad-Leaved Oaks (Quercus robur). Plos ONE 6(4):e17857.

Brown, J. S.; Kotler, B. P.; Smith, R. J. \& Wirtz II, W. O. 1988. The effect of owl predation on the foraging behavior of heteromyid rodents. Oecologia 76:408-415.

Cadotte, M. W.; Carscadden, K. \& Mirotchnick, N. 2011. Beyond species: functional diversity and the maintenance of ecological processes and services. Journal of Applied Ecology 48:1079-1087.

Clavel, J.; Julliard, R. \& Devictor, V. 2010. Worldwide decline of specialist species: toward a global functional homogenization. Frontiers in Ecology and the Environment 9(4):222-228.

CRACRAFT, J. 1985. Historical biogeography and patterns within the South American avifauna: Areas of endemism. Ornithological Monographs 36:49-84.

Daher, M. R. M.; Pereira, G. A.; Leal, S. R. M. \& Souza, D. S. 2013. As aves em fragmentos florestais de mata atlântica na bacia do rio Coruripe, Alagoas, p.79-115. In: Barros-Neto, G. G.; Fritscher, G. H. \& DAHER, M. R. M. orgs. Restauração do rio Coruripe - um projeto de resgate socioambiental. Maceió, Graziella Helena Fritscher. 160p.

DARIO, F. R. 2008. Estrutura trófica da avifauna em fragmentos florestais na Amazônia Oriental. Conscientiae Saúde 7:169-179.

DARIO, F. R. 2010. Avifauna em fragmentos florestais da Mata Atlântica no sul do Espírito Santo. Biotemas 23:105-115.

Donatelli, R. J.; Costa, T. V. V. \& Ferreira, C. D. 2004. Dinâmica da avifauna em fragmento de mata na Fazenda Rio Claro, Lençóis Paulista, São Paulo, Brasil. Revista Brasileira de Zoologia 21:97-114.

Farias, G. B. DE; Alves, A. G. C. \& Lins e Silva, A. C. B. 2007. Riqueza de aves em cinco fragmentos de Floresta Atlântica na Zona da Mata Norte de Pernambuco, Brasil. Biotemas 20:111-122.
Girao, L. C.; Lopes, A. V.; Tabarelli, M. \& Bruna, E. M. 2007. Changes in Tree Reproductive Traits Reduce Functional Diversity in a Fragmented Atlantic Forest Landscape. Plos One 2(9):e908.

Griffin, J. N.; MÉndez, V.; Johnson, A. F.; Jenkins, S. R. \& FogGo, A. 2009. Functional diversity predicts overyielding effect of species combination on primary productivity. Oikos 118:37-44.

Holland, S. M. 2003. Analytic Rarefaction 1.3. Available at $<\mathrm{http}: / /$ strata. uga.edu/ software/anRareReadme.html $>$. Accessed on 20 June 2017.

Hulme, P. E. \& Benkman, C. W. 2002. Granivory, p. 132-154. In: Herrera, C. \& Pellmyr, O. eds. Plant-animal interactions: An evolutionary approach. Oxford, Blackwell Science. 328p.

Ives, A. R. \& Dobson, A. P. 1987. Antipredator behavior and the populationdynamics of simple predator-prey systems. American Naturalist 130:431-227.

Jullien, M. \& Thiollay, J. M. 1996. Effects of rain forest disturbance and fragmentation: comparative changes of the raptor community along natural and human-made gradients in French Guiana. Journal of Biogeography 23:7-25.

Kramer-Schadt, S.; Kaiser, T. S.; Frank, K. \& Wiegand, T. 2011. Analyzing the effect of stepping stones on target patch colonization in structured landscapes for Eurasian lynx. Landscape Ecology 26:501513.

Lobo-Araújo, L. W.; Toledo, M. T. F.; Efe, M. A.; Malhado A. C. M.; Vital, M. V. C.; Toledo-Lima, G. S.; Macario, P.; Santos, J. G. \& LADLE, R. J. 2013. Bird communities in three forest types in the Pernambuco Centre of Endemism, Alagoas, Brazil. Iheringia, Série Zoologia 103:85-96.

Lovejoy, T. E.; Rankin, J. M.; BierregaArd, R. O. JR.; Emmons, L. H. \& VAN DER VOORT, M. E. 1984. Ecosystem decay of Amazon forest remnants. In: Nitecki, M. H. ed. Extinctions. Chicago, University of Chicago Press. 354p.

Lyra-Neves, R. M.; Dias, M. M.; AZeVedo Júnior, S. M.; Telino-Júnior, W. R. \& LarrazÁbal, M. E. L. 2004. Comunidade de aves da Reserva Estadual de Gurjaú, Pernambuco, Brasil. Revista Brasileira de Zoologia 21:581-592.

Magalhães, V. S.; Azevedo-Júnior, S. M.; Lyra-Neves, R. M.; TelinoJÚNIOR, W. R. \& SouZA, D. P. 2007. Biologia de aves capturadas em um fragmento de Mata Atlântica, Igarassu, Pernambuco, Brasil. Revista Brasileira de Zoologia 24:950-964.

Magurran, A. E. 1988. Ecological diversity and its measurement. London, Croom Helm. 192p.

MMA (Ministério do Meio Ambiente-BrasiL). 2014. Portaria no 444 de 17 de dezembro de 2014. Lista Oficial das Espécies da Fauna Brasileira Ameaçadas de Extinção. Available at $<$ http://pesquisa.in.gov.br/ imprensa/jsp/visualiza/ index.jsp?jornal $=1 \&>$ pagina $=121 \&$ data $=18 / 12 / 2014>$. Accessed on 26 July 2017.

Moraes, R. A.; Sawaya, R. J. \& Barrella, W. 2007. Composição e diversidade de anfíbios anuros em dois ambientes de Mata Atlântica no Parque Estadual Carlos Botelho, São Paulo, Sudeste do Brasil. Biota Neotropica 7:27-36.

Offerman, H. L.; Dale, V. H.; Pearson, S. M.; Bierregaard Jr., R. O \& O'NeILL, R. V. 1995. Effects of forest fragmentation on neotropical fauna: current research and data availability. Environmental Reviews 3:191-211.

Parker, T. A.; Stotz, D. F. \& FitzPatrick, J. W. 1996. Ecological and distributional databases. In: Stotz, D. F.; FitzPatrick, J. W.; PARKer, T. A. \& Moskovits, D. K. eds. Neotropical birds: ecology and conservation. Chicago, University of Chicago Press, p.113-436.

Pavoine, S.; Vallet, J.; Dufour, A. B.; Gachet, S. \& Daniel, H. 2009. On the challenge of treating various types of variables: application for improving the measurement of functional diversity. Oikos 118:391-402.

Pereira, G. A.; AraúJo, H. F. P. \& Azevedo-Júnior, S. M. 2016. Distribution and conservation of three important bird groups of the Atlantic Forest in north-east Brazil. Brazilian Journal of Biology 76:1004-1020.

Pereira, G. A.; Dantas, S. M.; Silveira, L. F.; Roda, S. A.; Albano, C.; Sonntag, F. A.; Leal, S.; Periquito, M. C.; Malacco, G. B. \& Lees, A. C. 2014. Status of the globally threatened forest birds of northeast Brazil. Papéis Avulsos de Zoologia 54:177-194.

Perfecto, I.; Vandermeer, J. H.; Bautista, G. L.; Nunez, G. I. \& GreENBERG, R. 2004. Greater predation in shaded coffee farms: the role of resident Neotropical birds. Ecology 85:2677-2681. 
Philpott, S. M.; Soong, O.; Lowenstein, J. H.; Pulido, A. L. \& Lopez, D. T. 2009. Functional richness and ecosystem services: bird predation on arthropods in tropical agroecosystems. Ecological Applications 19:1858-1867.

Piacentini, V. Q.; Aleixo, A.; Agne, C. E.; Maurício, G. N.; Pacheco, J. F.; Bravo, G. A.; Brito, G. R. R.; NaKa, L. N.; Olmos, F.; Posso, S.; Silveira, L. F.; Betini, G. S.; Carrano, E.; Franz, I.; Lees, A. C.; Lima, L. M.; Pioli, D.; Schunck, F.; Amaral, F. R.; Bencke, G. A.; Cohn-Haft, M.; Figueiredo, L. F. A.; Straube F. C. \& Cesari, E. 2015. Annotated checklist of the birds of Brazil by the Brazilian Ornithological Records Committee. Revista Brasileira de Ornitologia 23:91-298.

Piratelli, A. \& Pereira, M. R. 2002. Dieta de aves na região leste de Mato Grosso do Sul, Brasil. Ararajuba 10:131-139.

Prakash, V.; Pain, D. J.; Cunningham, A. A.; Donald, P. F.; Prakash, N.; Verma, A.; Gargi, R.; Sivakumar, S. \& Rahmani, A. R. 2003. Catastrophic collapse of Indian White-backed Gyps bengalensis and long-billed Gyps indicus vulture populations. Biological Conservation 109:381-390.

Purvis, A.; Gittleman, J. L.; Cowlishaw, G. \& Mace, G. M. 2000. Predicting extinction risk in declining species. Proceedings of Royal Society of London B: Biological Sciences 267:1947-1952.

R Core TEAM. 2014. R: A language and environment for statistical computing. R Foundation for Statistical Computing, Vienna, Austria. Available at <http://www.R-project.org/>. Accessed on 10 June 2014.

Ranta, P.; Blom, T.; Niemelã, J.; JoensuU, E. \& Sirtonen, M. 1998. The fragmented Atlantic Rain Forest of Brazil: size, shape and distribution of forest fragments. Biodiversity and Conservation 7:385-403.

Ribeiro, M. C.; Metzger, J. P.; Martensen, A. C.; Ponzoni, F. J. \& Hirota, M. M. 2009.The Brazilian Atlantic Forest: how much is left, and how is the remaining forest distributed? Implications for conservation. Biological Conservation 142:1141-1153.

Ridgely, R. S. \& Tudor, G. 1989. The birds of South America: the Oscines Passerines. v1. Austin, University of Texas Press. 596p.

Ridgely, R. S. \& Tudor, G. 1994. The birds of South America: the suboscines Passerines. v2. Austin, University of Texas Press. 940p.

RoDA, S. A. 2004. Composição e conservação de aves em ambientes fragmentados na Floresta Atlântica nordestina, Relatório Técnico CEPAN. Available at <http://www.cepan. org.br>. Accessed on 21 July 2016.

Roda, S. A. \& Pereira, G. A. 2005. Aves do Engenho Opinioso, Amaraji, Pernambuco. Relatório Técnico CEPAN. Available at $<\mathrm{http}: / / \mathrm{www}$. cepan.org.br 22p>. Accessed on 18 July 2016.

Roda, S. A. \& Pereira, G. A. 2006. Distribuição recente e conservação das aves de rapina florestais do Centro Pernambuco. Revista Brasileira de Ornitologia 14:331-344.

Rodrigues, R. C.; AraúJo, H. F. P.; Lyra-Neves, R. M.; Telino-Júnior, W. R. \& Botelho, M. C. N. 2007. Caracterização da avifauna na Área de Proteção Ambiental de Guadalupe, Pernambuco. Ornithologia 2:47-61.

SAurA, S.; Bodin, O. \& ForTin, M-J. 2014. Stepping stones are crucial for species' long-distance dispersal and range expansion through habitat networks. Journal of Applied Ecology 51:171-182.

SCHelhas, J. \& Greenber, R. 1996. Introduction: the value of Forest patches, p. 20-36. In: Schellas, J. \& Greenberg, R. eds. Forest patches in tropical landscapes. Washington, Island Press. 498p.

SchuchmanN, K. L. 1999. Family Trochilidae (Hummingbirds). In: DEL Hoyo, J.; Elliott, A. \& Sargatal, J. eds. Handbook of the birds of the world (Vol. 5): Barn-owls to Hummingbirds. Barcelona, Lynx Edicions. 759p.

Sekercioglu, C. H. 2006. Increasing awareness of avian ecological function. Trends in Ecology \& Evolution 21:464-471.
Sekercioglu, C. H.; Daily, G. C. \& Ehrlich, P. R. 2004. Ecosystem consequences of bird declines. Proceedings of the National Academy of Sciences of the United States of America 101:18042-18047.

SHAFFER, M. L. 1981. Minimum Population Sizes for Species Conservation. BioScience 31:131-134

Shimamoto, C. Y.; Botosso, P. C. \& Marques, M. C. M. 2014. How much carbon is sequestered during the restoration of tropical forests? Estimates from tree species in the Brazilian Atlantic Forest. Forest Ecology and Management 319:1-9.

Shultz, S.; Bradbury, R. B.; Evans, K. L.; Gregory, R. D. \& Blackburn, T. M. 2005. Brain Size and Resource Specialization Predict Long-Term Population Trends in British Birds. Proceedings of the Royal Society, B - Biological Sciences 272:2305-2311.

SICK, H. 1997. Ornitologia brasileira, 2ed. Rio de Janeiro, Nova Fronteira. $912 \mathrm{p}$.

Sigrist, T. 2009. Guia de Campo Avis Brasilis - Avifauna Brasileira: descrição das espécies. São Paulo, Avis Brasilis. 600p.

Silva, J. M. C. 1995. Birds of the Cerrado Region, South America. Steenstrupia 21:69-92.

Silva, J. M. C. \& Tabarelli, M. 2000. Tree species impoverishment and the future flora of the Atlantic Forest of northeast Brazil. Nature 404:72-74.

Silva, J. M. C.; Souza, M. C. \& Castelleti, C. H. M. 2004. Areas of endemism for passerine birds in the Atlantic forest, South America. Global Ecology and Biogeography 13:85-92.

Silveira, L. F.; Olmos, F. \& Long, A. J. 2003. Birds in Atlantic Forest Fragments in North-east Brazil. Cotinga 20:32-46.

Sodhi, N. S.; Didiuk, A. \& Oliphant, L. W. 1990. Differences in bird abundance in relation to proximity of Merlin nests. Canadian Journal of Zoology 68(5):852-854

SoKal, R. R. \& Michener, C. D. 1958. A statistical method for evaluating systematic relationships. University of Kansas Scientific Bulletin 28:1409-1438.

Telino-Jr, W. R.; Dias, M. M.; Azevedo Júnior, S. M.; Lyra-Neves, R. M. \& LARrazÁbal, M. E. L. 2005. Estrutura trófica da avifauna na Reserva Estadual do Gurjaú, Zona da Mata Sul, Pernambuco, Brasil. Revista Brasileira de Zoologia 22:962-973.

Terborgh, J.; Robinson, S. K.; Parker, T. A.; Munn, C. A. \& Pierpont, N. 1990. Structure and organization of an amazonian Forest bird community. Ecological Monographs 60:213-238.

TraYlor, M. A. \& FitzPATRICK, J. W. 1982. A survey of the tyrant flycatchers. The living Birds 19:7-50.

Uezu, A.; Beyer, D. D. \& Metzger, J. P. 2008. Can agroforest woodlots work as stepping stones for birds in the Atlantic forest region? Biodiversity Conservation 17:1907-1922.

Van Bael, S. A.; Philpott, S. M.; Greenberg, R.; Bichier, P. \& Barber, N. A. 2008. Birds as predators in tropical agroforestry systems. Ecology 89:928-934.

Vergara, P.; Jiménez, J. \& Schlatter, R. P. 2010. Effective point count duration for estimating bird species' richness in chilean forests. Zoological Studies 49:381-391.

Vielliard, J. M. E. \& Silva, W. R. 1990. Nova metodologia de levantamento quantitativo de avifauna e primeiros resultados no interior do Estado de São Paulo, Brasil. Anais do IV ENAV, Recife, Universidade Federal Rural de Pernambuco, p. 117-151.

Whelan, C. J.; Wenny, D. G. \& Marquis, R. J. 2008. Ecosystem services provided by birds. Annals of the New York Academy of Sciences 1134:25-60.

Wilcove, D. S.; Mclella, C. H. \& Dobson, A. P. 1986. Habita fragmentation in the temperate zone, p. 237-256. In: Soulé, M. E. ed. Conservation biology. The science of scarcity and diversity. Sunderland, Sinauer Associates. 584p.

WILLIS, E. O. 1979. The composition of avian communities in remanescent woodlots in southern Brazil. Papéis Avulsos de Zoologia 33:1-25. 